and secondary syphilis diagnoses increased on average $203.5 \%$ among blacks, $99.7 \%$ among Hispanics, and $43.7 \%$ among whites, and increases were observed in more areas for blacks (70\% of areas) than for Hispanics (44\% of areas) or whites (59\% of areas). (Abstract P1-S2.35 figure 1) The majority of areas (63\%) had increases in both HIV and syphilis in black men aged 13-24 years. Across area size strata, the youngest group of black men had the highest average percent increase in diagnoses of HIV and syphilis as well as the highest percentage of areas with increases in diagnoses.

Conclusions HIV and syphilis diagnoses have increased among young black men in almost all areas, suggesting that national trends are not driven by increases in a few large areas. Findings highlight the need for improved prevention efforts for young MSM, particularly young black MSM.

\section{P1-S2.36 PREVALENCE AND RISK FACTORS OF HSV-2 INFECTION AMONG MEN WHO HAVE SEX WITH MEN IN CHINA}

doi:10.1136/sextrans-2011-050108.93

S Chen, Y P Yin, H C Wang, W H Wei, A L Wang, Y Han, R R Peng, X S Chen, B X Wang. National Center for STD Control, China CDC, Nanjing, China

Background HIV prevalence among MSM is high, and epidemiological surveys have confirmed that HSV-2 infection can increase the risk of HIV infection and that HIV seroconversion is closely related to HSV-2 infection. In spite of high prevalence of HIV in MSM and strong association between HSV-2 infection and HIV seropositivity, there have been few studies about the prevalence of HSV-2 among MSM in China. To prevent the spread of HIV among this high risk population, there is an urgent need to investigate the HSV-2 prevalence and potential demographic and behavioural risk factors among MSM in China.

Methods A total of 1462 men who have sex with men were randomly selected from three regions (Changzhou, Guangzhou and Shenzhen) in China. Sera were tested to estimate the prevalence of HSV-2 (ELISA for the detection of HSV-2 type specific IgG) and HIV (ELISA for screening and WB for confirmation), while univariate and multivariate logistic regression analyses were used to estimate the degree of association between HSV-2 infection and potential demographic and behavioural risk factors. Results-The prevalence of HSV-2 antibodies was $16.0 \%$ (234 out of 1462). Factors independently associated with higher prevalence of HSV-2 antibodies were-age, educational level, exchange of sex for money and HIV status (see Abstract P1-S2.36 table 1).

Abstract P1-S2.36 Table 1 Multivariate analysis of risk factors associated with HSV-2 seropositivity among 1462 MSM in China

\begin{tabular}{lll}
\hline Characteristic & AOR (95\% CI) & p Value \\
\hline $\begin{array}{l}\text { Age } \\
\leq 30\end{array}$ & 1 & \\
$\quad>30$ & $1.988(1.426$ to 2.771$)$ & $<0.05$ \\
$\begin{array}{l}\text { Educational level } \\
\quad 0-9 \mathrm{yr}\end{array}$ & 2.247 & \\
$9-12 \mathrm{yr}$ & $1.479(1.520$ to 3.321$)$ & $<0.05$ \\
$>12 \mathrm{yr}$ & $1(1.020-2.144)$ & $<0.05$ \\
Exchange of sex for money & 1 & $<0.05$ \\
$\quad \begin{array}{l}\text { Sometimes } \\
\text { Never }\end{array}$ & $0.583(0.407$ to 0.835$)$ & \\
HIV status & & $<0.05$ \\
$\quad$ Positive & 2.763 & \\
$\quad$ Negative & $1(1.850$ to 4.128$)$ & \\
\hline
\end{tabular}

Conclusions The high prevalence of HSV-2 among MSM and strong association between HSV-2 infection and HIV seropositivity suggests the urgent need for intervention to prevent HSV-2 and HIV infections in high-risk MSM in China.

\section{P1-S2.37 INCIDENCE OF URETHRAL DISCHARGE SYNDROME AMING MEN WHO HAVE SEX WITH MEN AND ASSOCIATED RISK FACTORS IN INDIA; A COHORT ANALYSIS OF CLINIC ATTENDEES}

doi:10.1136/sextrans-2011-050108.94

${ }^{1} \mathrm{~A}$ Gurung, ${ }^{1} \mathrm{~A}$ Das, ${ }^{1}$ Prakash Naryanan, ${ }^{2} \mathrm{G}$ Neilson, ${ }^{3}$ Guy Morineau, ${ }^{2}$ Bitra George. ${ }^{1} \mathrm{FHI}$, New Delhi, India; ${ }^{2} \mathrm{FHI}$, Bankok, Thailand; ${ }^{3} \mathrm{FHI}$, APROThailand

Background Monitoring data from a cohort of men who have sex with men (MSM) attending 421 clinics located in six states of India from January 2004 to December 2009 were studied. These clinics were supported by Avahan (the India AIDS initiative of the Bill \& Melinda Gates Foundation) and provided services for sexually transmitted infections (STIs) including presumptive treatment for gonorrhoea and chlamydia; risk reduction counselling, treatment of STI syndromes and regular STI check-ups.

Methods Individual tracking data from the clinical forms were collected, merged and cleaned. Observed episodes of urethral discharge (UD) were counted as incident cases when clinical record prior to the episode reported no UD or if the patients had received the clinics' standard single dose directly observed treatment. The mid-point between visits was considered as the time of incident UD. Cox proportional hazard models were used to assess associations between incidence of UD and reported behaviours.

Results A total of 82690 MSM made 508469 visits to the clinics, constituting a cohort of 88458 person-years (median duration of follow-up 0.86 years; maximum, 5.9 years). 7292 cases of UD were considered as incident giving an incidence rate (IR) of 8.2 per 100 person years (PYs). The IR decreased from 82.7 per 100 PYs in 2005 to 2.8 per 100 PYs in 2009; the first year of follow-up having the highest incidence ( $H R=1.5, p<0.001)$. Factors influencing the risk of UD were-number of years in commercial sex; the first year being the most risky, $(\mathrm{HR}=4.3, \mathrm{p}<0.001)$; having more than ten clients per week $(\mathrm{HR}=1.6, \mathrm{p}<0.001)$; not using condoms at last sex $(\mathrm{HR}=2.3$, $\mathrm{p}<0.001)$; self-reported sexual identity of a penetrative role in anal sex $(\mathrm{HR}=1.4, \mathrm{p}<0.001)$, and not receiving treatment in the first three clinic visits, $(H R=2.8, p<0.001)$. Increasing frequency of clinic visits per year had a protective role $(\mathrm{HR}=0.9, \mathrm{p}<0.001)$. MSM visiting the clinics more than five times a year had an IR below 1 per 100 PY. While one-time presumptive treatment increased the risk of UD $(H R=3.2, p<0.001)$, there were no episodes of UD when the treatment was given at more than one consecutive visit.

Conclusion Urethral discharge syndrome among MSM shows a decline and could be a good proxy to include in future STI surveillance in resource-constrained scenarios. An analysis of MSM risk behaviour in India can help in risk profiling for targeted interventions.

\section{P1-S2.38 A SITUATIONAL ANALYSIS OF MSM AND HIV AT AAS'S CENTRE OASIS IN BURKINA FASO}

doi:10.1136/sextrans-2011-050108.95

${ }^{1} \mathrm{~T}$ A Soundiata, ${ }^{2} \mathrm{~K}$ Patrice, ${ }^{3} \mathrm{D}$ Elias, ${ }^{3} \mathrm{~T}$ Pascal, ${ }^{2} \mathrm{O}$ Filemon, ${ }^{2} \mathrm{~T}$ Issoufou. ${ }^{1}$ Association African Solidarite, Ouagadougou, Burkina Faso; ${ }^{2}$ A A S, Burkina Faso; ${ }^{3}$ A A S, Canada

Introduction In Burkina Faso at the end of 2008, the AIDS pandemic was characterised by a drop in HIV prevalence rates during the preceding years. In contexts in which a reduction in HIV prevalence is observed, the epidemic tends to be concentrated among certain 
groups considered to be highly vulnerable to infection, including MSM. This present study was conducted to answer key questions with a view to gaining a better understanding of the reality of MSM with regard to HIV/AIDS at AAS. The results are to contribute to the development of an appropriate response strategy.

Methodology The study's aim was to identify possible paths of action and strategies pertaining to MSMs' access to STI/AIDS care, treatment and prevention services at AAS. The study was based on a questionnaire focusing on MSMs' contextual environment and the socio-behavioural factors that determine their vulnerability to STIs and HIV.

Principle Results The questionnaire was administered among 84 MSM at the Centre Oasis, at places where they gather or at their homes. Of those men, 13\% are between 17 and 19 years old; $33 \%$ between 20 and 24; 25\% between 25 and 29; and $29 \%$ age 30 and over. $78 \%$ are single; $5.2 \%$ have a girlfriend; and $16.8 \%$ are married to a woman. Survey participants' first sexual experience was with an MSM friend in $70.5 \%$ of cases; with an unknown partner in $18.3 \%$ of cases; and with classmates in $11.2 \%$ of cases. $89.4 \%$ of the MSM surveyed consented to their first sexual encounter with another man; their motivation was-sexual desire (68\%); curiosity (12\%); need of money (11\%); need of a service $(9 \%) .47 \%$ used a condom during that first sexual encounter, from which 13\% emerged with injuries and bleeding. $81 \%$ of those surveyed maintain parallel sexual relationships with women. $83 \%$ are aware of HIV; 69\% gonorrhoea; $61 \%$ syphilis; and they do not master the other STIs. $97 \%$ know that unprotected sex is high-risk; $83.4 \%$ know about relevant risks from contaminated objects, and $58.1 \%$ are familiar with relevant risks from blood transfusions. $61.1 \%$ systematically use condoms during sex with other men.

Conclusion This exploratory study has allowed us to gain a number of insights. We will now use this data as a basis for improving the services we offer to MSM.

Abstract P1-S2.38 Table 1 Incidence rates of urethral discharge among MSM coming into the Avahan clinics

\begin{tabular}{|c|c|c|c|c|c|}
\hline Characteristics & $\begin{array}{l}\text { IR per } 100 \text { PY } \\
\text { Incidence rate } \\
\text { per } 100 \\
\text { person years }\end{array}$ & $\begin{array}{l}95 \% \mathrm{Co} \\
\text { Lower }\end{array}$ & Upper & HR & p Value \\
\hline \multicolumn{6}{|l|}{ Typology (Self-identity) } \\
\hline Kothi (Receptive) & 6.4 & 6.1 & 6.6 & \multicolumn{2}{|l|}{ Reference } \\
\hline Double Decker (Versatile) & 7.3 & 6.9 & 7.6 & & \\
\hline Panthi (Penetrative) & 14.5 & 14 & 15.2 & 1.04 & $<0.001$ \\
\hline \multicolumn{6}{|c|}{ Number of years in commercial sex } \\
\hline$<5$ years & 5 & 4.5 & 5.6 & \multicolumn{2}{|l|}{ Reference } \\
\hline $2-5$ years & 4.6 & 4.1 & 5.2 & & \\
\hline$>1$ year & 14.9 & 14.5 & 15.3 & 4.29 & $<0.001$ \\
\hline \multicolumn{6}{|l|}{ Number of clients per week } \\
\hline$<5$ clients per week & 2.8 & 2.5 & 3.2 & \multicolumn{2}{|l|}{ Reference } \\
\hline $6-10$ clients per week & 3.6 & 3.1 & 4 & & \\
\hline$>10$ clients per week & 6.7 & 5.9 & 7.6 & 1.62 & $<0.001$ \\
\hline \multicolumn{6}{|l|}{ Self-reported condom use } \\
\hline Condom used in last sex & 4.3 & 4.1 & 4.4 & \multicolumn{2}{|c|}{ Reference } \\
\hline No condom used in last sex & 15 & 14.5 & 15.4 & 2.28 & $<0.001$ \\
\hline \multicolumn{6}{|c|}{ Cefixime and azithromycin (Presumptive treatment-PT given in the first three clinic visits) } \\
\hline Given PT & 7.9 & 1.7 & 1.9 & \multicolumn{2}{|c|}{ Reference } \\
\hline Not given PT & 11.1 & 20.8 & 21.9 & 2.79 & $<0.001$ \\
\hline \multicolumn{6}{|c|}{ Number of visits per year to the clinic } \\
\hline More than 8 visits per year & 0.5 & 0.4 & 0.6 & \multicolumn{2}{|l|}{ Reference } \\
\hline $7-8$ visits per year & 0.8 & 0.6 & 0.9 & & \\
\hline $5-6$ visits per year & 0.9 & 0.7 & 1 & & \\
\hline $3-4$ visits per year & 1.3 & 1.28 & 1.5 & & \\
\hline $1-2$ visits per year & 27.6 & 27 & 28.3 & 0.85 & $<0.001$ \\
\hline
\end{tabular}

\section{P1-S2.39 CHARACTERISTICS OF LYMPHOGRANULOMA VENEREUM (LGV) INFECTION AMONG HOMOSEXUAL MEN IN MELBOURNE.}

doi:10.1136/sextrans-2011-050108.96

\section{Bissessor. Melbourne Sexual Health, Carlton, Australia}

Background Lymphogranuloma venereum (LGV) has re-emerged among men who have sex with men (MSM) internationally. Previous cases among MSM in Australia have been reported. We aimed to characterise a series of LGV cases seen at the Melbourne Sexual Health Centre.

Methods We reviewed all cases of LGV seen at the Melbourne Sexual Health Centre between 2005 and 2010. During this period MSM who were diagnosed with rectal chlamydia using strand displacement assay were routinely questioned about symptoms of proctitis using a symptom check list. Those with symptoms of proctitis had specimens forwarded for Chlamydia trachomatis omp1 genotyping. Genotyping of chlamydia-positive first void urine and penile ulcers in MSM was undertaken on selected MSM based on clinical presentation.

Results During the study period, of 292 chlamydia positive anorectal specimens that were genotyped, $21(7.2 \%)$ tested positive for LGV. Of the eight chlamydia positive urine or penile specimens genotyped, four were positive for LGV. There was also one case of an inguinal bubo that tested LGV positive from aspirated pus. In all cases L2 or L2b type was isolated. Of the 25 cases of LGV, 18 (72\%) were in HIV positive men. Of the 21 men who had ano-rectal LGV, all had ano-rectal symptoms, namely-ano-rectal pain (71\%), anal discharge (62\%) and/or rectal bleeding (52\%). All but one of the LGV infected men received at least a 3 -week course of doxycycline $100 \mathrm{mg}$ twice daily. Two men with ano-rectal LGV remained LGV positive on follow-up when tested 3 months later. Both these patients were noted to have had unprotected sex following initial treatment, hence, it is unclear whether this was due to treatment failure or reinfection.

Conclusions LGV continues to be seen among MSM in Melbourne presenting with proctitis. Genotyping of anal chlamydia infections has been useful in distinguishing LGV from the more common nonLGV chlamydial infections, therefore ensuring appropriate antibiotic therapy and follow-up are instigated.

\section{P1-S2.40 HIV AND SYPHILIS COINFECTION AMONG MEN WHO HAVE SEX WITH MEN, 34 STATES, USA-2009}

doi:10.1136/sextrans-2011-050108.97

J Su. Centers for Disease Control and Prevention, Atlanta, USA

Background During the early 2000s, reports of the re-emergence of syphilis among men who have with men (MSM) in the USA described a high prevalence of HIV coinfection in different localities, often among middle-aged men. Since 2001, syphilis has increased among men in younger age groups. Here, we present a description of HIV coinfection among MSM of different races and ethnicities with primary and secondary syphilis across multiple states during 2009. Methods We reviewed data reported to CDC from states in the USA that reported HIV coinfection status for at least $70 \%$ of cases of primary and secondary $(P \& S)$ syphilis during 2009. These data originated from $\mathrm{P} \& \mathrm{~S}$ syphilis patient interviews and included census region, sex, sex of sex partner, race, ethnicity, and HIV infection status. Prevalence of coinfection was calculated using coinfected patients as the numerator, and all $\mathrm{P} \& \mathrm{~S}$ syphilis patients who were asked about their HIV status as the denominator.

Results 34 states and Washington, DC from all regions of the USA met inclusion criteria, accounting for $82 \%$ of all $\mathrm{P} \& \mathrm{~S}$ syphilis in the 